is a workable alternative approach and it relies on two fundamental factors:

- In dental practice the vast majority of dental patients are booked as 'elective' patients. We know who they are, we know when they are coming and we know what we are planning to do for them when they arrive. This means we can plan in advance of treatment and we can choose to delay treatment if necessary. Medical personnel in ICUs at hospitals have to be in close proximity to infectious patients - we do not have to be, nor should we be

- The only patients who present any form of risk from dental treatment (AGPs included) are those patients who are actively infectious at the time of treatment. Someone who has not been infected by the virus poses zero risk and someone who has been infected but recovered and seroconverted poses zero risk. Only a very small percentage of the population are infectious at any one time and these individuals are infectious only for a limited time.

The smart strategy means we need to identify the risk patients by advance swab testing of our patients when they require an appointment. Swab testing could be done onsite by a trained practice member in advance of any appointment booking. Those who test negative would be allowed normal full access to dental services. These appointments would be undertaken using exactly the same protocols as were used prior to the COVID-19 outbreak. Those who test positive will need to delay booking until they have a negative test - presumably about four weeks later if they remain well.

The main advantage of this approach is that it is proportional, specific and minimally disruptive. Furthermore, if the dental profession begins to test all of our patients this will have a significant advantage for the population as a whole, taking a burden away from the government or other healthcare sectors.

Therefore, we do not need to change dentistry fundamentally forever, we need to be SMART and TARGETED for now. If testing is done by the dental profession there will be spin-off benefits for all. To use the current 'management speak' it is a win/win situation.

J. A. Woodcock, Chalfont St Giles, UK https://doi.org/10.1038/s41415-020-1800-7

\section{A new era for dental education}

Sir, while going through a large pile of past copies of the $B D J$, a front cover image is shown of a 'Face-mask for the protection of the dentist while operating' circa 1920, 100 years ago (Fig. 1).

It is important to reflect that we have always been an infection aware profession and have therefore often been at the forefront of infection control in the surgery for both our patients and the whole dental team. ${ }^{1}$ Along with other dental schools, ${ }^{2}$ we stopped student patient contact before lockdown, however, we have continued with online lectures, tutorials, one to ones and imaginative online remote assessment. We are now grappling with the expectation of teaching again in September. Naturally, much important education can occur for example, with group work on evidence-based dentistry and with teaching preclinical skills in skills laboratories. Indeed, some elements of education, such as case reports, clinical reasoning and team care planning are probably easier to timetable across year groups and inter-professionally in the virtual environment, than face to face.

As primarily a school teaching dental nurses to certificate level, separate degree programmes in dental therapy and in dental hygiene, as well as teaching final year students from King's College London integrated team care, our focus on minimal

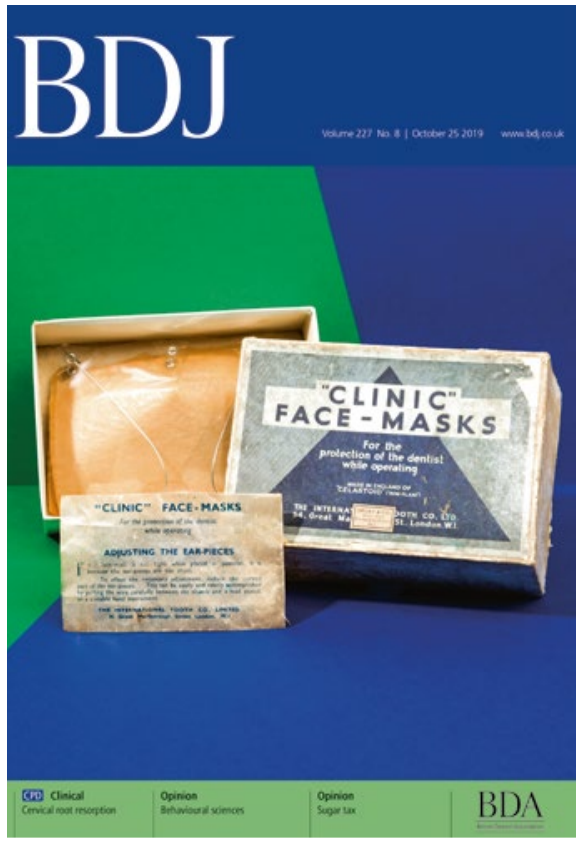

Fig. 1 The cover image of the BDJ Volume 227 issue 8 , published on 25 October 2019 intervention comes to the fore in a post COVID-19 era.

However, even behind our FFP3 masks and visors, the logistics of teaching clinical skills and caring for patients will remain a significant but critical challenge in our large open clinics with narrow passageways between clinical units. In addition, the need for one to one qualified dental nursing and new equipment that produces less aerosol, will not just need imagination, but like all of high street dentistry, considerable financial investment. We are about to enter a new era of dental education.

\section{R. Radford, C. Louca, Portsmouth, UK}

\section{References}

1. Gordon B L, Burke F J T, Bagg J, Marlborough H $S$, McHugh E S. Systematic review of adherence to infection control guidelines in dentistry. J Dent 2001; 29: 509-516.

2. Coulthard P. Dentistry and coronavirus (COVID-19)Moral decision-making. Br Dent J 2020; 228: 503-505.

https://doi.org/10.1038/s41415-020-1801-6

\section{Diagnosing OSCC via video}

Sir, we would like to highlight a case of oral squamous cell carcinoma (OSCC) brought to our attention via digital and virtual communications methods. A 78-year-old male, in the midst of the recent pandemic lockdown, could not access an in-person consultation at his local GP surgery. His son was able to arrange tele-communications with the GP using a smartphone to take photos at the patient's home and email them to the GP who, after review, forwarded these via email to our Oral and Maxillofacial Surgery Department for assistance (Fig. 1).

On receiving the photos an immediate video consultation was set up between clinician, patient and family member using NHS Attend Anywhere to allow an initial history and assessment to be undertaken. It was suspected that the lesion was sinister and further higher quality photographs were requested and received reinforcing concern of an advanced lower lip SCC.

Urgent head and neck scans and biopsy investigations were organised for a one-time hospital visit, to reduce the number of in-person interactions for this shielded patient. By this stage a primary care assessment, secondary care referral, subsequent history and examination and planning of one-day further investigations 